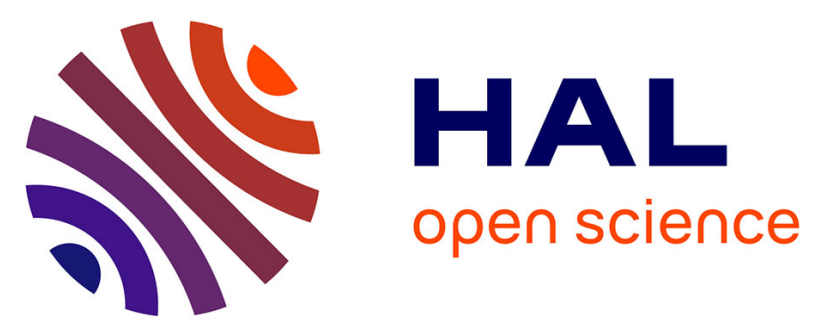

\title{
APPLICATION DE LA MÉTHODE DES ÉLÉMENTS FINIS À LA MODÉLISATION DE STRUCTURES PÉRIODIQUES UTILISÉES EN ACOUSTIQUE
}

Anne-Christine Hladky, R. Bossut, J. Decarpigny, C. Audoly

\section{- To cite this version:}

Anne-Christine Hladky, R. Bossut, J. Decarpigny, C. Audoly. APPLICATION DE LA MÉTHODE DES ÉLÉMENTS FINIS À LA MODÉLISATION DE STRUCTURES PÉRIODIQUES UTILISÉES EN ACOUSTIQUE. Journal de Physique Colloques, 1990, 51 (C2), pp.C2-399-C2-402. 10.1051/jphyscol:1990294 . jpa-00230717

\section{HAL Id: jpa-00230717 https://hal.science/jpa-00230717}

Submitted on 1 Jan 1990

HAL is a multi-disciplinary open access archive for the deposit and dissemination of scientific research documents, whether they are published or not. The documents may come from teaching and research institutions in France or abroad, or from public or private research centers.
L'archive ouverte pluridisciplinaire HAL, est destinée au dépôt et à la diffusion de documents scientifiques de niveau recherche, publiés ou non, émanant des établissements d'enseignement et de recherche français ou étrangers, des laboratoires publics ou privés. 
COLLOQUE DE PHYSIQUE

Colloque C2, supplément au $\mathrm{n}^{\circ} 2$, Tome 51, Février 1990

ler Congrès Français d'Acoustique 1990

APPLICATION DE LA MÉTHODE DES ELEMENTS FINIS À LA MODÉLISATION DE STRUCTURES PERIODIQUES UTILISÉES EN ACOUSTIQUE

\author{
A.C. HENNION, R. BOSSUT, J.N. DECARPIGNY et C. AUDOLY* \\ Iaboratoire d'Acoustique, URA 253 CNRS, Institut Supérieur \\ d'Electronique du Nord, 41 Boulevard Vauban, F-59046 Lille Cedex, \\ France \\ * Groupe d'Etude et de Recherche de Détection Sous-Marine, D.C.A.N. \\ Toulon, Le Brusc, F-83140 Six Fours les Plages, France
}

Résumé: Un modèle mathématique tridimensionnel a été développé pour analyser la diffraction d'une onde plane acoustique par un réseau dont la périodicité est à une ou deux dimensions. Il repose sur la méthode des éléments finis et exploite le code ATILA. Ce papier décrit les résultats obtenus pour différents types de réseaux. La comparaison à des résultats analytiques antérieurs ou à des mesures permet une excellente validation du modèle.

Abstract: A three-dimensional mathematical model has been developed to analyze the scattering of a plane acoustic wave from simple or doubly periodic structures. It relies upon the finite element method and uses the ATILA code. This paper describes results obtained for the scattering of a plane wave from different gratings. Comparison between finite element results and previous analytical or experimental results demonstrates the accuracy of the model.

\title{
Introduction.
}

La diffraction d'une onde acoustique par un réseau dont la périodicitê est à une ou deux dimensions est susceptible de nombreuses applications, notamment en acoustique sous-marine: réseaux de tubes compliants, mono ou multicouches, enrobés ou non đ'un matériau viscoélastique, structures multicouches à inclusions périodiques, du type Alberich, revêtements anécholques... Du point de vue conception, il faut noter toutefois que, si de nombreux modeles analytiques ou semi-analytiques ont permis de décrire le comportement de réseaux simples, tels que des réseaux de cylindres circulaires, de tubes compliants de section elliptique /1-4/ ou de tubes de section rectangulaire et allongée /5. $6 /$, ces modèles requièrent souvent des développements algébriques importants qui réduisent leur utilisation à des géométries particulières. Aussi, 1 'analyse de réseaux plus complexes du fait de leur géometrie, des matériaux qui les constituent, de la direction d'incidence de $I^{\prime}$ onde ou des modes de vibration concernes, nécessite toujours une approche numérique. La modélisation de structures périodiques à 1 'aide du code éléments finis ATILA /7/ pernet d'atteindre cet objectif. Dans cette présentation, le formalisme mathematique est tout d'abord decrit pour une structure tridimensionnelle. Ensuite quelques exemples tests permettant de démontrer la précision de la méthode sont présentés. Enfin, la généralité de celle-ci est illustrée à l'aide de plusieurs applications des structures complexes de reseaux de tubes compliants ou de materiaux a inclusions périodiques, pour une onde acoustique d'incidence quelconque.

\section{Formulation theorique}

\section{I.1. Modè le mathematique général.}

Le formalisme mathématique nécessaire à la description de structures périodiques est présenté pour un domaine d’êtude tridimensionnel, la périodicité étant à deux dimensions (Figure 1). Le domaine est décomposé en trois régions successives par deux plans parallèles au plan de la structure diffractante, notés $\mathrm{S}^{+}$et $\mathrm{S}^{-}$. Les première et troisième régions sont des domaines fluides semi-infinis, dans lesquels le champ de pression est développé en série d'ondes planes progressives et évanescentes. Dans la premiêre région, 


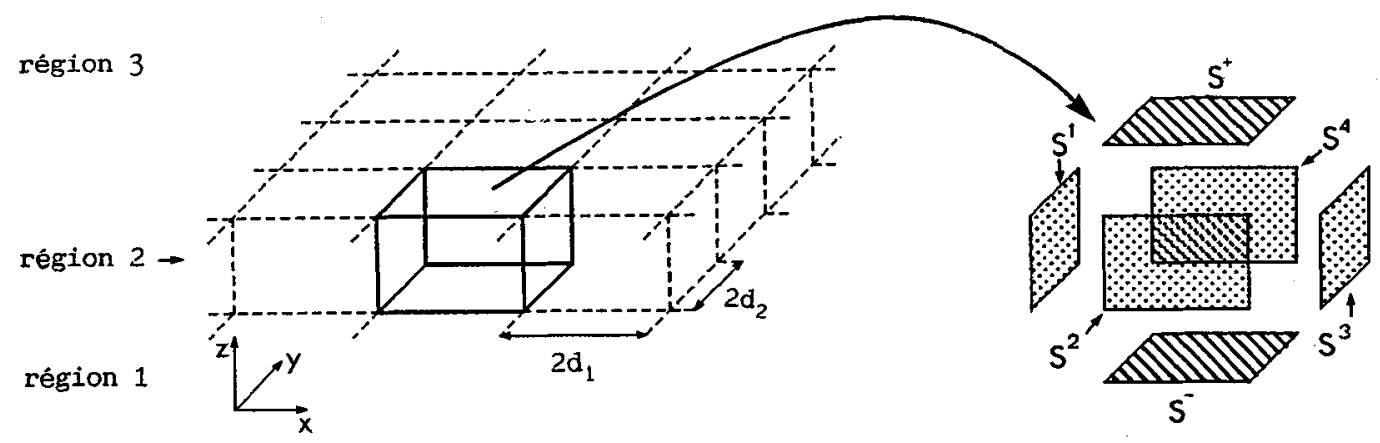

Figure 1: Structure périodique dans deux directions.

1'une des ondes progressives est $1^{\prime}$ 'onde Incidente. La seconde région comprend les structures diffractantes périodiques et une partie du fluide environnant. Dans cette région, la double périodicité du problème peut être prise en compte à l'aide de conditions cycliques du type Bloch-Floquet, c'est à dire d'une relation de phase spécifique entre des points distants d'un nombre entier de fois le pas du réseau. L'étude de cette région peut ainsi être réduite à une maille élémentaire, qui fait l'objet d'un maillage par éléments finis et les relations de Bloch-Floquet, appliquées aux valeurs du déplacement ou de la pression sur des noeuds séparés d'une période, sont utilisées pour définir les conđitions aux limites entre des cellules élémentaires voisines, sur les plans $S^{1}, S^{2}, S^{3}$ et $S^{4}$. Par ailleurs, les équations de continuité pour le champ de pression et sa dérivée normale sont écrites sur la frontière entre les régions 1 et 2 d'une part, 2 et 3 d'autre part.

\section{I.2. Application de la méthode des éléments finis.}

Les relations de Bloch-Floquet permettent de réduire la modélisation de la région II à une cellule élêmentaire, qui est ensuite maillée en utilisant les éléments finis /8/. Classiquement, les équations du système fournissent le champ de déplacement et le champ de pression. Les relations de Bloch-Floquet permettent des combinaisons linéaires de certaines lignes et colonnes de ce système, i.e. la condensation des degrés de liberté appartenant aux faces $S^{3}$ et $S^{4}$ limitant la cellule elémentaire. La prise en compte de l'effet des domaines fluides externes introduit des relations matriclelies entre les valeurs nodales de la dérivée normale de la pression réfléchie (respectivement transmise) et les valeurs nodales de la pression réfléchie (respectivement transmise). Lorsque la structure diffractante est infinie dans une direction, le probleme est bidimensionnel et des simplifications évidentes sont réalisées $/ 9 /$.

\section{Résultats.}

Utilisant la méthode des éléments finis décrite ci-dessus, les variations du coefficient de transmission en fonction de la fréquence peuvent être étudiées. Le coefficient de transmission est défini par $20 \mathrm{Log}\left(\mathrm{P}_{t r} / \mathrm{P}_{1}\right)$ où $\mathrm{P}_{\mathrm{tr}}$ et $\mathrm{P}_{1}$ sont les pressions transmise et incidente. Pour les configurations utilisées ici, les fréquences sont choisies pour qu'une seule onde progressive se propage de part et d'autre du réseau, dans la même direction que l'onde incidente, les autres ondes étant évanescentes au sens où elles n'ont une influence que dans le champ proche. Ainsi, le coefficient de transmission se calcule en utilisant uniquement le terme d'ordre zéro dans le développement de la pression en série d'ondes planes.

\section{1. Réseaux de cylindres circulaires.}

Il s'agit d'étudier la diffusion d'ondes sonores par un réseau de cylindres circulaires en PVC, I'onde plane d'excitation étant en incidence normale. Les pertes dans le PVC sont choisies égales à $6 \%$. Les résultats du modèle éléments finis, incluant et 
excluant les pertes, sont comparés à des mesures /4/. La figure 2 montre un bon accord entre ces courbes. Le réseau de tubes étant ensuite enrobé de polyuréthane, la flgure 3 compare les résultats du modèle éléments finis aux mesures. L'accord est bon entre ces courbes. L'écart observe a $13 \mathrm{kHz}$ est probablement da aux difficultés rencontrés pour connaltre avec précision les propriétes du polyuréthane, qui varient en fonction de le fréquence et de la température.
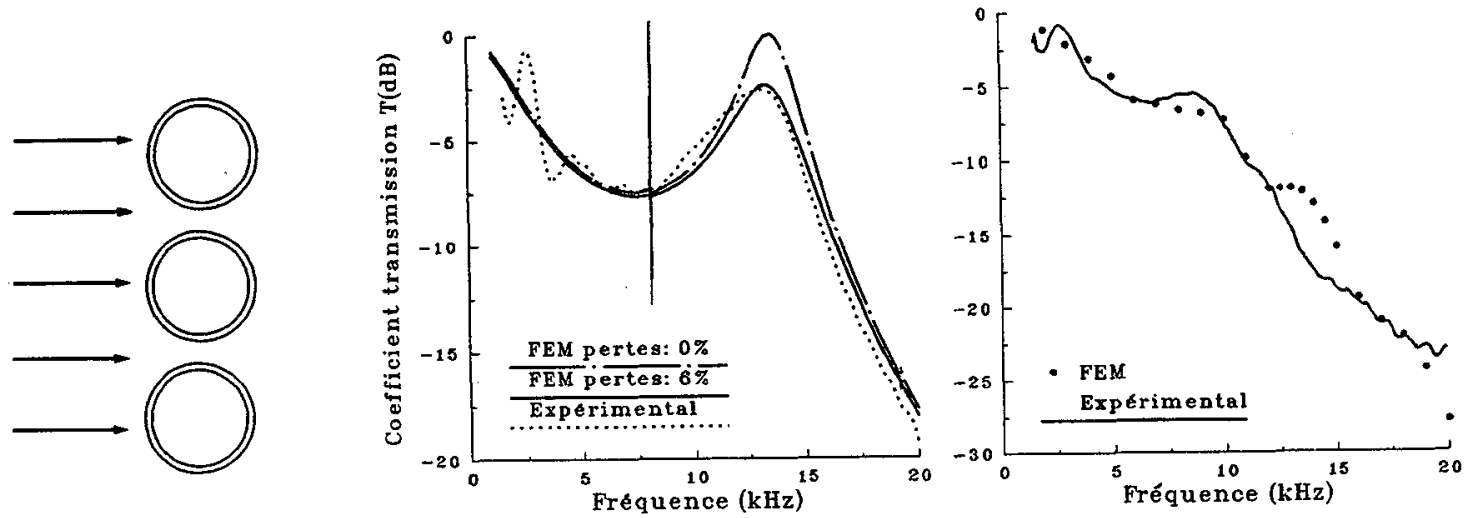

Cylindres circulaires: Figure 2: Réseau immergé; Figure 3: Réseau enrobé de polyuréthane

II. 2. Résecux de tubes compliants multicouches.

L'intérêt de ces réseaux est considérable. Le recouvrement des bandes de fréquence dans lesquelles les sous-réseaux produisent une réflexion importante permet de réaliser des réflecteurs très large bande. Un tel réseau est decrit à la figure 4 . Les tubes, de section rectangulaire, sont assimilés, d'un point de vue mécanique, a deux plaques parallèles encastrées. Ces tubes, infinis dans une direction, forment un réseau de pas $2 d$. La figure 4 montre également la variation avec la fréquence du coefficient de transmission pour un
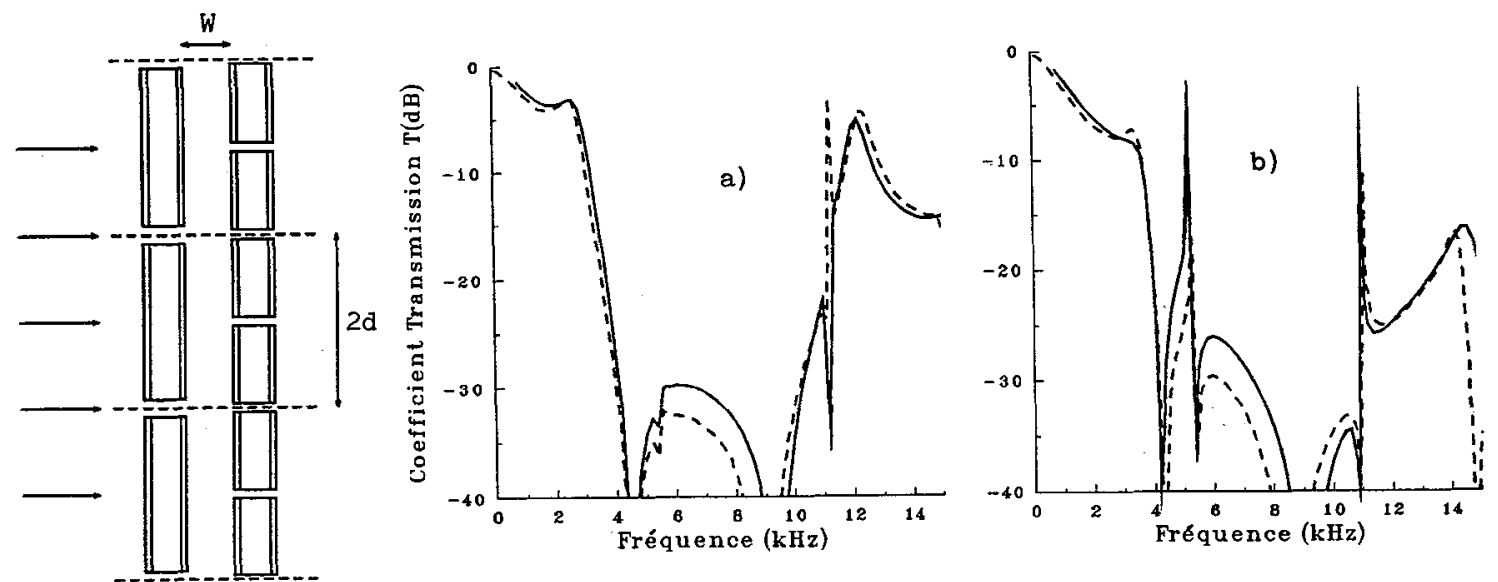

Figure 4: Réseau de tubes compliants double couche: a): $W / d=6 ; b): W / d=0.85$ Trait plein: résultats éléments finis, trait pointillé: résultats analytiques $/ 4 /$ 
espacement entre les couches de $W / d=6$ puis de $W / d=0.85$. Les résultats du modèle éléments finis sont comparés à des résultats analytiques, utilisant un modele de guide d'ondes $/ 4,6 /$. L'accord est parfalt entre les deux méthodes. Lorsque 1 'espacement entre les deux couches diminue, le champ de pression au voisinage des tubes est moins uniforme et l'interaction du champ proche tend a modifier les performances en termes de largeur de bande de rejection.

\section{II.3. Réseaux de type Alberich.}

Il s'agit d'une plaque de résine silicone contenant des inclusions cylindriques d'air, qui forment un réseau périodique à deux dimensions (Figure 5). Les proprietés de la résine, en fonction de la frequence d'excltation et de la température, sont relevées à partir de courbes maitresses. La figure 5 compare les coefficients de transmission de la plaque, calculé par éléments finis d'une part, mesuré d'autre part. L'accord est bon entre les deux courbes. Le premier pic est da à l'excitation d'un mode de résonance de la cavité.
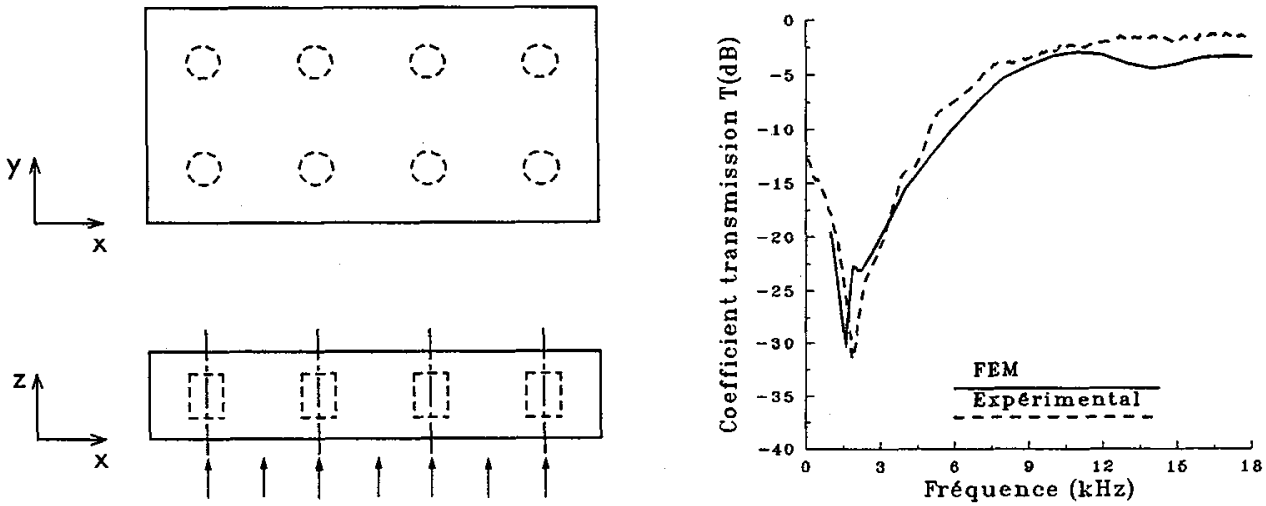

Figure 5: Panneau de type Alberich

Conclusion.

Cette analyse du comportement acoustique de réseaux à périodicité simple ou double a prouvé la capacité et la souplesse du code ATILA. Un bon accord a été trouvé entre les résultats obtenus par éléments finis, par des méthodes analytiques ou par la mesure. L'un de nos objectifs est maintenant d'étendre cette méthode à la modélisation de structures ou de matériaux à périođicité tridimensionnelle. Elle permettrait ainsi de déterminer, par exemple, les proprietés homogénétsées de ces matériaux, dans la limite des grandes longueurs d'onde.

\section{Références.}

11/E. BURKE, V. TWERSKY, IEEE Trans. Antennas Propag., AP-14, 465-480 (1966).

/2/G.A. BRIGHAM, J.J. LIBUHA, R.P. RADLINSKI, J .Acoust. Soc. Am. . 61, 48-59, $(1977)$.

/3/C. AUDOLY, G. DUMERY, a paraitre dans Acustica, (1989).

/4/C.AUDOLY, "Etude de barrières acoustiques formées de réseaux d'obstacles résonnants". Thèse de Doctorat, Untversite de Toulon et du Var (1989).

$15 / \mathrm{V}$. VOVK, V.T. GRINCHENKO, L.A. KONONUCHENKO, Akust. Zh., 22, 201-205, (1976) [Sov. Phys. Acoust. , 22, 113-115, (1976)].

/6/R.P. RADLINSKI, M.M. SIMON, J. Acoust. Soc. Am., 72, 607-614, (1982).

$/ 7 / J . N$. DECARPIGNY, J.C. DEBUS, P. TIERCE, B. TOCQUET, D. BOUCHER, J. Acoust. Soc. Am. , 78, 1499-1507, (1985).

18/0.C. ZIENKIEWICZ, "The finite element method", Ed. Mac Graw Hill, (1977).

/9/A.C. HENNION, R. BOSSUT, J.N. DECARPIGNY, C. AUDOLY, à paraître dans le J. Acoust. Soc. Am. (1989). 